\title{
Effect of Molecular Weight on Electro Spun Pcl Based Composite Fibrous Mats
}

\author{
TS Sampath Kumar ${ }^{*}$, V Yogeshwar Chakrapani ${ }^{1,2}$, Pooja Vardhini Natesan ${ }^{1}$, Deepa K Raj ${ }^{2}$ and TV Kumary \\ ${ }^{1}$ Department of Metallurgical and Materials Engineering, Indian Institute of Technology, India \\ ${ }^{2}$ Tissue Culture Laboratories, Sree Chitra Tirunal Institute for Medical Sciences and Technology, India
}

Received: September 09, 2017; Published: September 21, 2017

*Corresponding author: TS Sampath Kumar, Medical Materials Laboratory, Department of Metallurgical and Materials Engineering, Indian Institute of Technology Madras, Chennai-600036, India, Email: tssk@iitm.ac.in

\begin{abstract}
Controlling the biodegradation of scaffolds to tune with that of the growing tissue is crucial for tissue regeneration. Although molecular weight of a polymer is known to influence its degradation, such studies have not been reported for electrospun Polycaprolactone (PCL) based composite scaffolds. In the present study, two low molecular weight PCL, 10 and $45 \mathrm{kDa}$ PCL (10PCL and 45PCL) have been analyzed for their electrospun ability and biodegradation in comparison with $80 \mathrm{kDa}$ PCL (80PCL) in forming pure polymeric and hydroxyapatite composite nanofibrous mats. The 45PCL and 80PCL were electro spin able but the 10PCL resulted in electro spraying of particles. However, 45PCL needed a higher concentration (20 wt.\%) of polymer than the 80PCL ( $8 \mathrm{wt} . \%$ ) in order to electrospin bead free uniform fibres. The fibres obtained were in the diameter range of $310 \pm 50 \mathrm{~nm}$ and $400 \pm 100 \mathrm{~nm}$ for the $45 \mathrm{PCL}$ and $45 \mathrm{PCL} / \mathrm{HA}$ composite mats respectively. The Hydroxyapatite (HA) incorporation in the composites was confirmed through X-ray diffraction and spectroscopic methods. The 45PCL/HA composite degrades at a relatively faster rate than the 80PCL/HA composite. The electrospun mats were also found to be non cytotoxic (Live/Dead assay), commendable cellular metabolic activity (MTT assay) and good proliferation of osteoblast like cells (HOS) as evidenced through fluorescent and scanning electron microscopy. This work demonstrated the feasibility of fabricating PCL based electrospun composite scaffolds having controlled degradation rates.
\end{abstract}

Keywords: Electrospinning; Low molecular weight; Controlled degradation; Bone tissue engineering

Abbreviations : PCL: Poly Capro Lactone; ECM: Extra Cellular Matrix; HA: Hydroxy Apatite; MW: Molecular Weight; HOS: Human Osteo Sarcoma; HV: High voltage; ATR : Attenuated Total Reflection

\section{Introduction}

Electro spinning is commonly utilized for forming nanofibrous scaffolds, to mimic the extra cellular matrix (ECM), for tissue engineering applications. Polycaprolactone (PCL) is often favored for Electrospinning of tissue engineering scaffolds. One of the reasons for its widespread usage is PCL's ability to degrade in physiological conditions, by hydrolysis of its ester linkages [1], into intermediates such as 6-hydroxylcaproic acid and acetyl coenzyme A, which are eliminated from the body through the citric acid cycle [2]. However, PCL degrades very slowly due to the five hydrophobic $\mathrm{CH} 2$ moieties in its repeating units (Figure 1) [3].

This has been the reason for denying the 'ideal polymer' status for PCL in orthopedic applications in spite of its promising results [4]. Methods to improve its degradation include blending with hydrophobic polymers [5], adding ceramic fillers and plasma treatment [6]. Hydroxyapatite (HA) is the most commonly studied ceramic filler used for fabrication of PCL based composites for bone tissue engineering due to its similarity to the natural bone mineral through which properties such as bioactivity, osteoconductivity and osteoinductivity can be imparted to the electrospun scaffolds. HA can also provide a buffering effect for the acidic by-products of polymer degradation [7].

It is well known that molecular weight (MW) influences the kinetics of degradation. Higher MW has larger chain lengths, thereby necessitating more number of ester bonds to be cleaved for subsequent degradation [8]. Studies on PCL implants have observed a reduction in the MW of the starting material, only after which fragmentation of the low MW PCL occurs $[9,10]$. Generally PCL with a MW of $80 \mathrm{kDa}$ or higher is used for Electrospinning and studies on low MW PCL (LMWPCL) are very few and not comprehensive in nature $[11,12]$. This may be due to the difficulty in forming an electrospinnable solution using the LMWPCL polymer. In Electrospinning, the polymer chain entanglements serve to stabilize the jet and form continuous fibres [13]. 
Below a critical concentration or number of chain entanglements (entanglement density), brittle fibres, heavily beaded fibres or particles are formed [14]. In a polymer solution both concentration (and volume fraction) (C) and MW affect the entanglement density [14]. In the current study, two LMWPCLs, namely 10 and $45 \mathrm{kDa}$ (10PCL and 45PCL) have been analyzed for their electrospinnability by comparing it with the typically electrospun $80 \mathrm{kDa}$ PCL (80PCL). Additionally composite scaffolds have been electrospun by incorporating HA particles in the electrospinnable PCL solutions. The electrospun scaffolds were characterized for their physicochemical properties and their degradation behaviours were compared. The cytotoxicity and cytocompatibility of the scaffolds were analyzed through Live/Dead and MTT assay while the growth and proliferation of human osteosarcoma (HOS) cells on the scaffolds were analyzed using fluorescent microscopy and scanning electron microscopy.

\section{Materials and Methods \\ Materials}

PCL (10, 45 and $80 \mathrm{kDa})$, lipase enzyme and cell culture reagents were purchased from Sigma Aldrich, USA. Unless otherwise mentioned all chemicals were purchased from MERCK, India. HA was synthesized by a microwave assisted method locally as reported earlier [15].

\section{Electrospinning}

An 8 wt. \% solution of 80PCL was prepared using chloroform and methanol (3:1). Various concentrations of 45PCL (10-20 wt. \%) and 10PCL (15-30 wt. \%) were prepared using the same solvent system and tested for electrospinnability. To the final electrospinnable concentrations, known amounts of microwave synthesized nano HA were added, so as to obtain different PCL/HA ratios (95/5, $92 / 8,90 / 10$ ) and stirred for $48 \mathrm{~h}$ to form the composite solutions. Electrospinning was carried out using an in house developed Electrospinning setup comprising a high voltage (HV) unit (Model 2-A, Zeonics Systech, India), syringe pump (NE1000, New Era Pump Systems Inc., USA) and a grounded metallic collector. Solutions were loaded into a $10 \mathrm{ml}$ syringe fitted with a blunted 22 gauge needle. The anode of the HV unit was connected to the needle tip. The metallic collector was covered with an aluminum foil on which the electrospun mats were collected. The ratio resulting in best electrospun composite mats was used for further studies. For comparison purposes a similar ratio was prepared using 80PCL and HA. Microscope cover slips, cleaned with ethanol, were placed at random positions over the collector for collecting samples for cell culture studies.

\section{Scanning Electron Microscopy (Sem) And Energy Dispersive X-Ray Analysis (Eds)}

The electrospun mats were sputter coated (Gatan, USA) with gold and analyzed using a SEM (FEI- Quanta 400, Netherlands) at an accelerating voltage of $10 \mathrm{kV}$. The images were analyzed using image processing software, Digimizer@. Fibre diameter was measured randomly at 50 places in each image and averaged. Additionally the elemental composition of the samples were also analyzed using EDS attached to the SEM.

\section{X-Ray Diffraction (Xrd) Analysis}

The electrospun mats and HA were analyzed for their crystallinity and sample composition in an X-ray diffractometer (D8 DISCOVER, Bruker, USA) with $\mathrm{Cu}$ K $\alpha$ radiation $(\lambda=1.54 \AA$ ) at a scanning speed of $0.1^{\circ} /$ step and scanning rate of 1 step per second. The peaks of HA were indexed according to JCPDS file no. 9-432.

\section{Fourier Transform Infrared (Ft-Ir) Spectroscopy}

The electrospun mats were analyzed for their functional groups within the spectral range of 4000-510 cm-1 at a resolution of 4 cm-1 using a FT-IR spectroscopy (Spectrum Two, Perkin- Elmer, USA) fitted with an attenuated total reflection (ATR) attachment.

\section{Contact Angle Analysis}

The hydrophilic natures of the electrospun mats were assessed by evaluating the contact angle made with a drop of double distilled water on them using a contact angle measuring instrument (Easy Drop, KRUSS, and Germany). Averages of 5 measurements were taken from triplicates of each sample.

\section{Degradation Studies}

The enzymatic degradation of the electrospun mats was carried out in a $0.1 \mathrm{M}$ phosphate buffer solution (PBS) with lipase enzyme at a concentration of $1 \mathrm{mg} / \mathrm{ml}$ and $0.02 \%$ sodium azide as a bacteriostatic agent for 6 weeks. $2 \times 2 \mathrm{~cm} 2$ samples were cut from the mats, weighed (WI) and incubated at $37^{\circ} \mathrm{C}$ in $20 \mathrm{ml}$ of the solution. Samples were removed every week from the solution, rinsed with deionized water and gently pressed between two filter papers to remove excess water before being dried in a vacuum desiccator and weighed to obtain the dry weight (WD). The weight loss was calculated using the formula Weight loss (\%) $=\{(\mathrm{WI}-\mathrm{WD})$ $/ \mathrm{WI}\} \times 100$ the change in $\mathrm{pH}$ of the PBS solution as the degradation proceeded was also assessed every week using a pH meter (Eutech pH 700, Eutech Instruments, Singapore)

\section{Cell Culture Studies}

The cytotoxicity and cytocompatibility of electrospun mat was evaluated by cell adhesion studies and MTT assay respectively. Briefly human osteosarcoma (HOS) cells were sub cultured and seeded to electrospun mat at a density of $1 \times 104$ cells $/ \mathrm{cm} 2$ and incubated for $48 \mathrm{~h}$. After incubation the cells seeded mat was evaluated by live dead staining, actin-phalloidin staining, SEM and MTT assay. Test materials were sterilized with $70 \%$ ethanol for $10 \mathrm{~min}$ and subsequently washed thrice with culture medium containing serum.

\section{Live/Dead Assay}

In order to evaluate the cytotoxic response of materials, the viability of the cells on the test samples, was assessed using Live/ Dead assay. Briefly, cells at a concentration of $1 \times 104$ cells $/ \mathrm{cm} 2$ were seeded on the test materials and glass cover slips (control), and incubated for $48 \mathrm{~h}$. Subsequently the cells were stained with fluorescein diacetate [FDA $(10 \mu \mathrm{g} / \mathrm{ml})$ (Sigma Aldrich, India)] for $15 \mathrm{~min}$ and non viable cells were counter stained with propidium iodide [PI $(10 \mu \mathrm{g} / \mathrm{ml})$ (HiMedia, India)] for $1 \mathrm{~min}$. The cells were visualized under a fluorescence microscope (DMI 6000E, Leica, Germany) using filters I3/N21. 


\section{Actin-Phalloidin Staining}

Cells cultured on test materials and control glass cover slip were evaluated by rhodamine phalloidin (Invitrogen, USA) staining. After $48 \mathrm{~h}$ the cell monolayer was washed thrice with phosphate buffered saline (PBS) and fixed in $4 \%$ par formaldehyde for $2 \mathrm{~h}$. The fixed cells were rinsed with PBS, and permeabilised with $0.1 \%$ triton X-100 in PBS for 3 min. Subsequently triton X-100 was removed by rinsing thrice with PBS. Rhodamine conjugated with phalloidin (1:1000) was added to the cells and incubated for $15 \mathrm{~min}$. This was followed by counter staining nucleus with Hoechst 33258 for 1 min. Cells were visualized under a fluorescence microscope (DMI 6000 E, Leica) using filters N21/A.

\section{SEM Analysis}

For SEM analysis of cell adhesion, samples were fixed by $2.5 \%$ glutaraldehyde in phosphate buffer followed by rinsing with PBS. After rinsing, the samples were dehydrated in graded concentration of ethanol and gold sputter coated before proceeding for SEM (FEIQuanta 400, Netherlands) imaging.

\section{MTT Assay}

The cell activity was calculated by quantifying the metabolic activity through MTT [3-(4,5- dimethylthiazol-2-yl)-2,5diphenyltetrazolium bromide] assay. The test materials after 48 $\mathrm{h}$ of cell adhesion were analyzed by MTT assay. Samples were rinsed thrice with medium and $400 \mu \mathrm{l}$ of MTT solution (1 mg/ $\mathrm{ml}$ MEM without serum) was added and incubated for $2 \mathrm{~h}$. After $2 \mathrm{~h}$ the formazan crystals formed were solubilised by $800 \mu \mathrm{l}$ of isopropanol and transferred to a 96 well plate. The optical density (OD) of the solution at $570 \mathrm{~nm}$ was measured using a UV/Visible spectrophotometer (Power Wave XS, Biotek, US). Cell activity was calculated as the percentage relative to the untreated control cells using the equation, Cell activity $(\%)=(O D$ test cells $/ O D$ control $) \times$ 100. (1)

\section{Statistical analysis}

Statistical significance was determined using Student's t test. P-values less than 0.05 were considered as statistically significant.

\section{Results}

\section{Electrospinning}

The various concentrations (wt. \%) of 45PCL and 10PCL that were attempted to be electrospun are shown in Table 1. It was observed that the regularly reported concentration of 8-12 wt. \% for Electrospinning 80PCL was not sufficient for Electrospinning of both 45PCL and 10PCL. Although 45PCL formed bead free fibres around $20 \mathrm{wt}$. \%, 10PCL was not electrospinnable up to even 30 $w \mathrm{t} \%$, beyond which the solution was not process able. Hence 10PCL was not used for the Electrospinning of composite mats. The $20 \mathrm{wt}$. $\%$ of 45 PCL was used for the forming the composite mats. A 45PCL/ HA of $90 / 10$ was found to be the ideal ratio for Electrospinning of the composite mat. The optimized and electrospun 45PCL and 45PCL/HA mats will be referred to as LPCL and LPCLHA while the electrospun 80PCL and 80PCL/HA mats will be referred to as PCL and PCLHA respectively (Table 1).
Table 1: Various Polymer Concentrations, Flow Rate, Voltage of 45PCL and 10PCL.

\begin{tabular}{|c|c|c|c|c|c|}
\hline S.No. & Polymer & $\begin{array}{c}\text { Polymer } \\
\text { Concentration } \\
\text { (Wt. \%) }\end{array}$ & $\begin{array}{l}\text { Flow } \\
\text { rate } \\
(\mathrm{ml} / \mathrm{h})\end{array}$ & $\begin{array}{c}\text { Voltage } \\
(\mathrm{kV})\end{array}$ & Result \\
\hline 1. & & 10 & $\begin{array}{c}0.5- \\
2.0\end{array}$ & $8-15$ & $\begin{array}{c}\text { Particles } \\
\text { and } \\
\text { extremely } \\
\text { beaded } \\
\text { structures }\end{array}$ \\
\hline 2. & 45PCL & 15 & $\begin{array}{c}0.5- \\
1.5\end{array}$ & $10-18$ & $\begin{array}{c}\text { Less } \\
\text { particles } \\
\text { and beaded } \\
\text { fibrous } \\
\text { structures }\end{array}$ \\
\hline 3. & & 20 & $\begin{array}{c}0.5- \\
1.0\end{array}$ & $12-20$ & $\begin{array}{l}\text { Bead free } \\
\text { uniform } \\
\text { fibres }\end{array}$ \\
\hline 4. & & 15 & $\begin{array}{c}0.5- \\
1.0\end{array}$ & $10-15$ & Particles \\
\hline 5. & 10PCL & 20 & $\begin{array}{c}1.0- \\
2.0\end{array}$ & $15-18$ & $\begin{array}{c}\text { Larger } \\
\text { particles } \\
\text { with non } \\
\text { uniform } \\
\text { structures }\end{array}$ \\
\hline 6. & & 25 & $\begin{array}{c}0.5- \\
2.0\end{array}$ & $18-22$ & $\begin{array}{c}\text { Large } \\
\text { globules } \\
\text { of polymer } \\
\text { with } \\
\text { few } \\
\text { particles }\end{array}$ \\
\hline
\end{tabular}

\section{SEM and EDS Analysis}

The surface morphologies of the electrospun mats as observed under a SEM are shown in (Figure 1). It was observed that LPCL and LPCLHA had fibres in the range of $310 \pm 50 \mathrm{~nm}$ and $400 \pm 100$ $\mathrm{nm}$, while PCL and PCLHA had larger diameter fibres in the range of $550 \pm 120$ and $640 \pm 165 \mathrm{~nm}$ respectively. In both molecular weight regimes, the effect of HA seems to be the same in increasing the overall diameter of the fibres. This is generally observed in Electrospinning of composites [16]. The corresponding EDS patterns are shown as inset in the images. Incorporation of HA 
in the composite mats is evidenced by the presence of $\mathrm{Ca}$ and $\mathrm{P}$ elements (Figure 1).

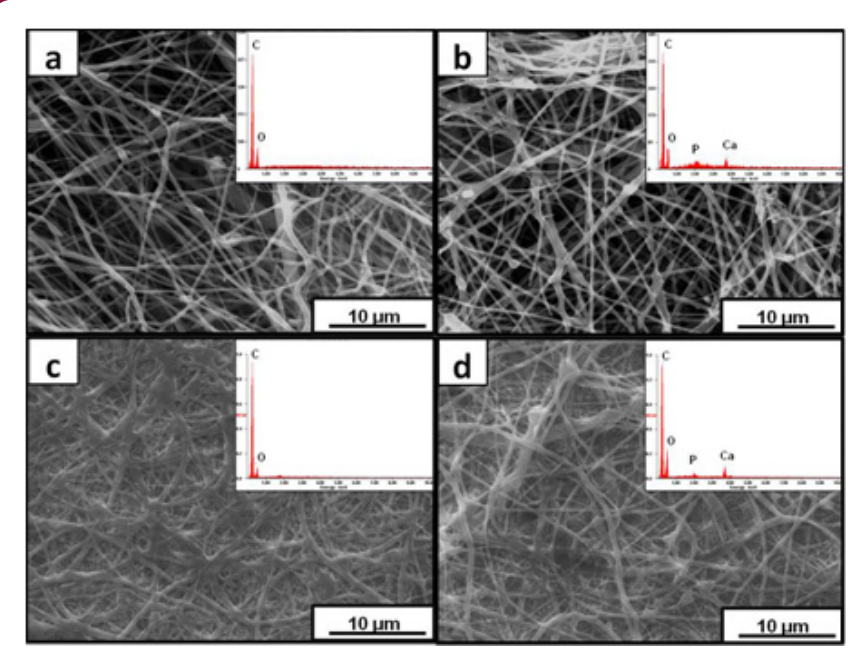

Figure 1: SEM images of surface of a) LPCL b) LPCLHA c) PCL and d) PCLHA. The corresponding EDS patterns are shown in the respective insets.

\section{XRD}

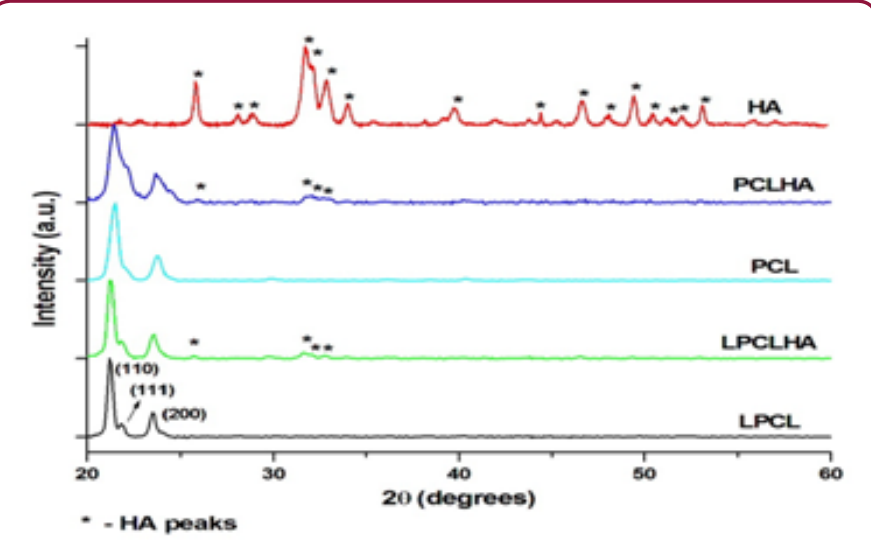

Figure 2: XRD patterns of the electrospun mats and HA.

The XRD patterns of the electrospun mats and HA are shown in (Figure 2). Crystalline PCL has an orthorhombic crystal structure with XRD peaks around $21.7^{\circ}$ and $23.7^{\circ}$ along with a smaller shoulder at $22^{\circ}$ corresponding to the (110), (200) and (111) planes. These were observed for all the electrospun mats. The pattern obtained for HA was indexed according to JCPDS file no. 9-432. The high intensity peaks of HA were visible in the patterns obtained for the composite mats, thus confirming their presence in the fibers (Figure 2).

\section{FT-IR}

The FT-IR spectra of the electrospun mats and that of HA are shown in (Figure 3). All the electrospun mats display infrared bands related to the stretching modes, namely asymmetric $\mathrm{CH} 2$ stretching (2923 cm-1), symmetric CH2 stretching (2857 cm-1), carbonyl stretching (1720 cm-1), C-O and C-C stretching in the crystalline phase (1293 cm-1) and asymmetric COC stretching (1240 cm-1) corresponding to PCL. CH2 bending vibrations at 1464, 1418, 1397 and $1366 \mathrm{~cm}-1$ were also observed. The spectra of HA displays all functional groups characteristic of HA [15]. In both the composite mats, the vibration bands corresponding to (PO4)3-bending at 1023 and $560 \mathrm{~cm}-1$ of HA were also observed. Thus, the FT-IR spectra confirm the functional groups of the polymer and ceramic as well as the incorporation of HA in the composites (Figure 3).

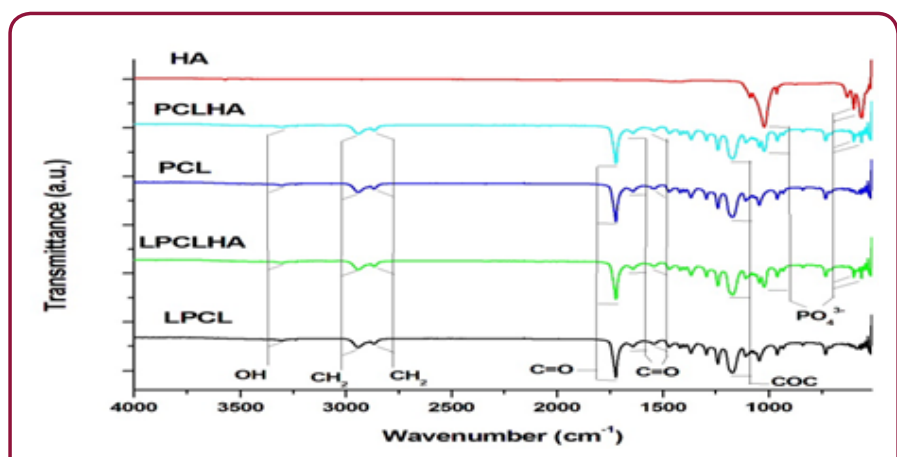

Figure 3: FT-IR spectra of the electrospun mats and HA.

\section{Water contact angle}

Figure 4 shows the results of contact angle analysis performed on the electrospun mats. All the contact angles are obtuse due to the hydrophobic nature of PCL. It is interesting to see that the contact angles on the composite mats are lesser when compared to their polymer counterparts (Figure 4).

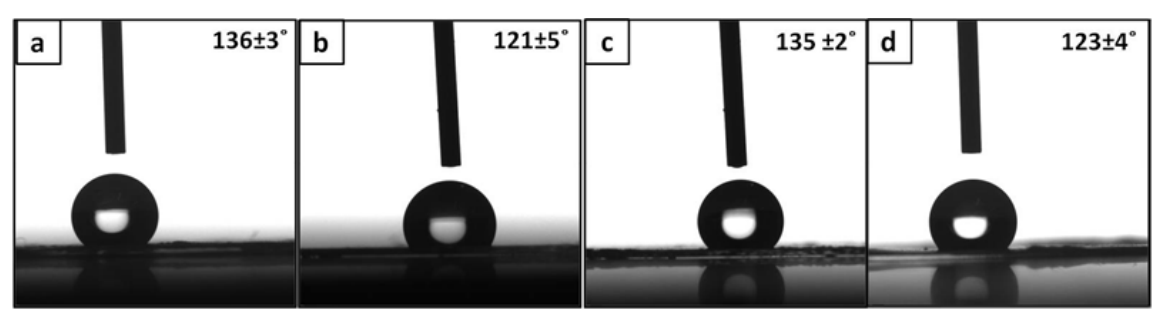

Figure 4: Images of a water droplet on a) LPCL b) LPCLHA c) PCL and d) PCLHA. The contact angle made by the droplet is also mentioned in the image.

\section{Degradation studies}

The weight loss with time is shown in Figure $5 \mathrm{a}$. It is seen that LPCL and LPCLHA degrade at a faster rate than PCL and PCLHA respectively. Thus, the presence of HA has accelerated the degradation process. Though initially PCLHA degrades slower than LPCL, after 2nd week it degrades faster than LPCL. PCL electrospun mat maintained its structure, although with considerable weight loss, even after 6 weeks. LPCLHA displayed complete disintegration 
after 4th week, while PCLHA and LPCL lost their integrity after 5th week. The variation in $\mathrm{pH}$ of the PBS containing the electrospun sample mats is shown in Figure 5b. All samples show continuous decrease in $\mathrm{pH}$ with the increase in time. While LPCL shows the lowest pH by the end of 6 weeks, PCLHA shows the highest $\mathrm{pH}$. Initially $\mathrm{pH}$ decrease in PCL is not as prominent as in LPCL. However after 2 nd week, $\mathrm{pH}$ decreases more rapidly for PCL. This can be linked to the slower degradation of PCL compared to LPCL. Even though there is rapid degradation in PCLHA after 2nd week, the corresponding change in $\mathrm{pH}$ is not abrupt. By 4th week, the composites have a less acidic $\mathrm{pH}$ than the polymer samples which maybe a consequence of HA particles released due to degradation of the mats (Figure 5).
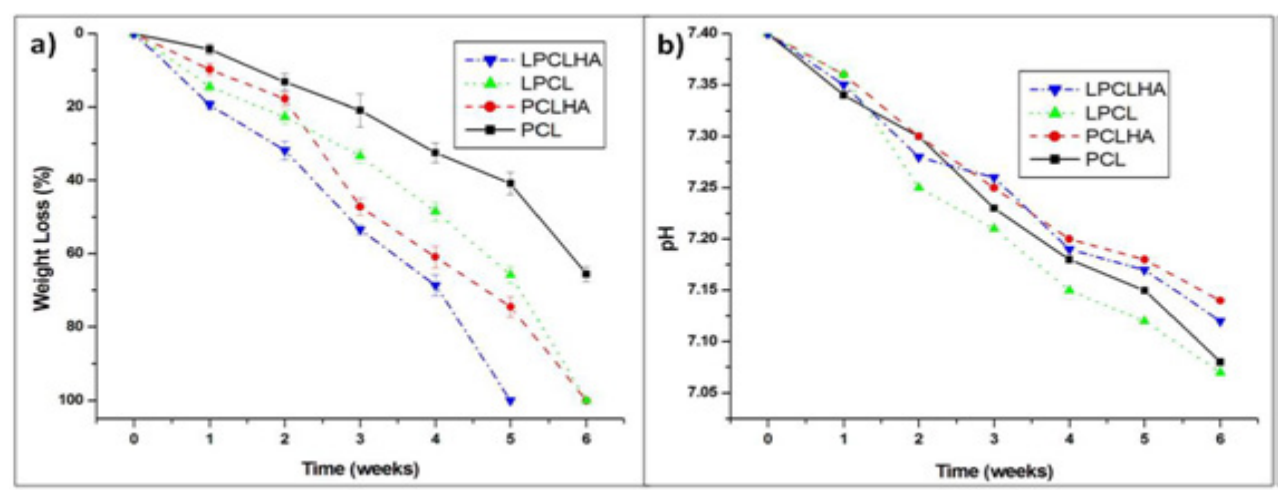

Figure 5: Weight loss (a) and change in $\mathrm{pH}$, (b) with time during degradation.

\section{Cell Culture studies}

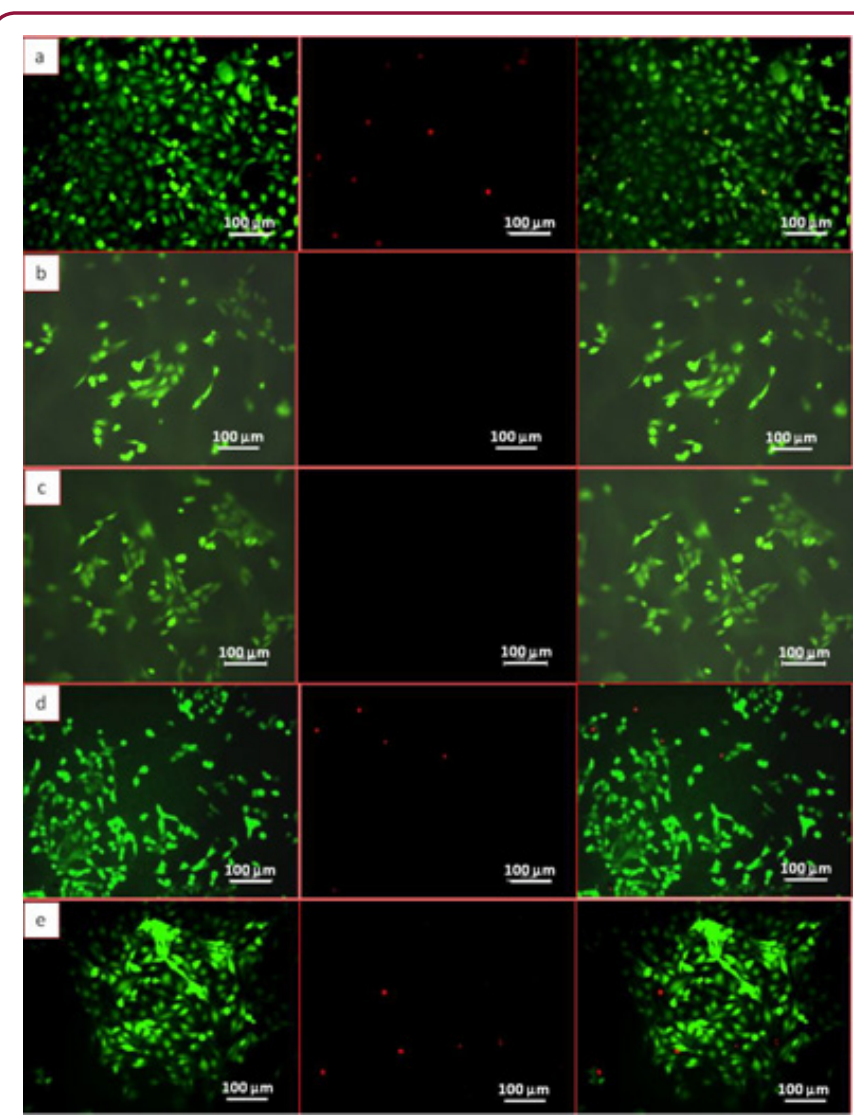

Figure 6: Live/Dead image of (a) control coverslips (b) LPCL (c) LPCLHA (d) PCL and (e) PCLHA. First image represents fluorescence from live cells second shows signal from dead cells and third is the merge of first and second images.

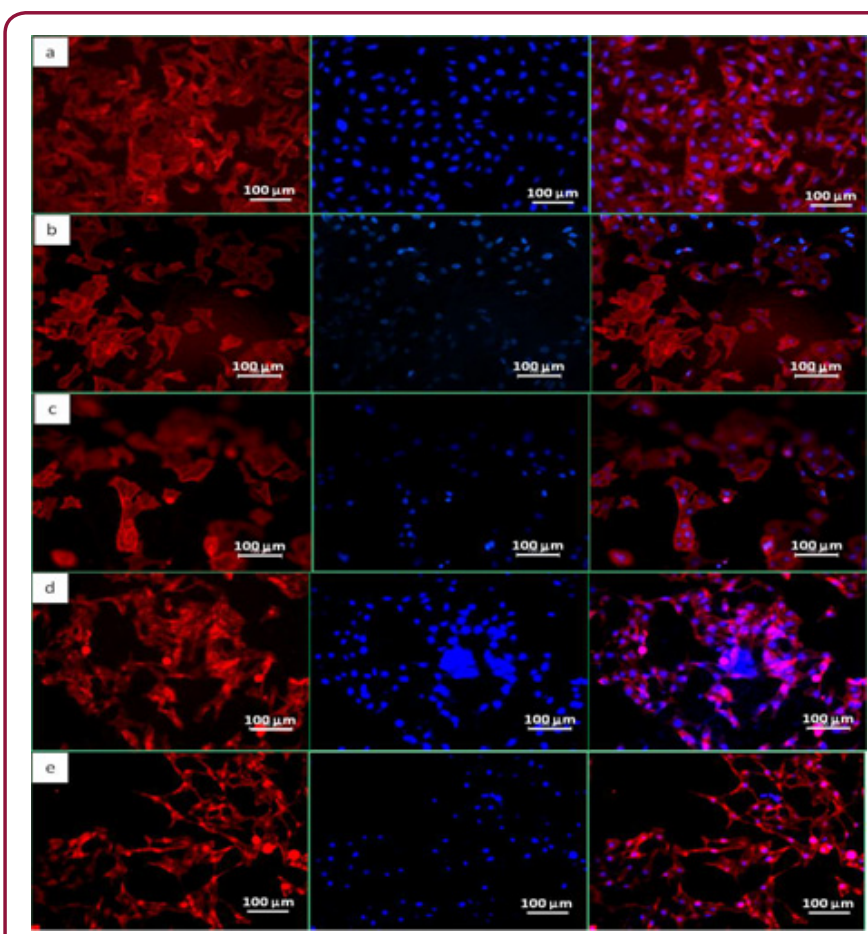

Figure 7: Actin phalloidin image of (a) control cover slips (b) LPCL (c) LPCLHA (d) PCL and (e) PCLHA. First image represents fluorescence from actin, second shows signal from nucleus and third is the merged images.

Fluorescence images of the HOS cell populations on the control and test samples, with the cytoplasm of the live cells stained with FDA (green), the nucleus of the dead cells stained with PI (red) and the respective overlay, are shown in (Figure 6). The Live/ Dead assay distinguishes live cells by the presence of intracellular 
esterase activity by hydrolysis of FDA. After $48 \mathrm{~h}$ of incubation, a high density of live cells was revealed on all the samples, depicting the non cytotoxic nature of the materials (Figure 6). The fluorescent microscope images of the stained HOS cells adhered on the electrospun mats are shown in (Figure 7). The rhodamine stained actin filaments and the Hoechst 33258 stained nucleus were visible in the control and test samples. While the cells seem to be well grown and adhered on all the samples, the cells on LPCL and LPCLHA seem to be a more spread out than on PCL and PCLHA mats (Figure 7). Figure 8 shows the SEM analysis of the cells adhered on the electrospun mat. HOS cells were adhered and spread well on all the materials implying that the electrospun mats support cell growth (Figure 8). Cytocompatibility was evaluated by MTT assay. The cell metabolic activity normalized with cell control is shown in (Figure 7). It is seen that all the electrospun mats display a metabolic activity of $100 \%$ or more. LPCLHA shows the maximum metabolic activity and is also significantly higher than the rest of the samples (Figure 9).

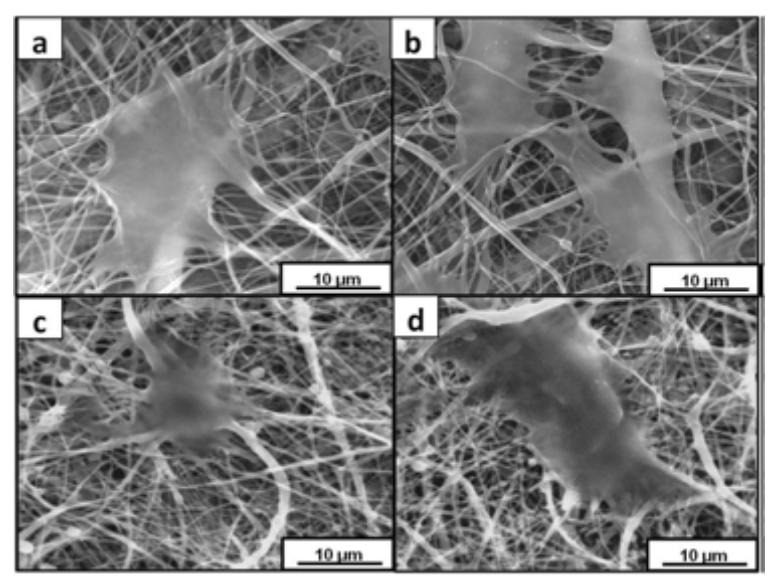

Figure 8: SEM images of the cells proliferated on a) LPCL b) LPCLHA c) PCL and d) PCLHA mats.

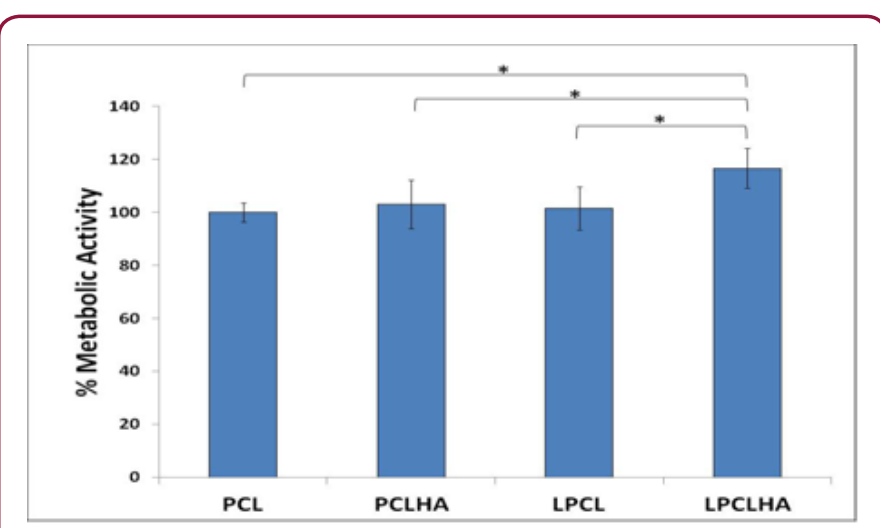

Figure 9: Cell metabolic activity measure on the electrospun samples.

\section{Discussion}

The 45PCL concentration necessary to form an electrospinnable solution was higher (20 wt. \%) than what is reported for 80PCL (812 wt. \%). In general Electrospinning of a polymer solution occurs when there are enough chain entanglements [14]. Also for a given MW entanglement density increases with increasing concentration. Hence it can be said that the critical entanglement density necessary for Electrospinning the 45PCL was achieved at $20 \mathrm{wt} \%$. For preparing the composite mat, it was seen that a HA wt. \% of more than 10 resulted in beaded fibres. Moreover LPCL solution results in fibres of diameters lesser than PCL and PCLHA respectively. It may be said that even when using a higher concentration, a solution made of lower MW polymer results in finer fibres than when using a higher MW of the same polymer. A detailed study of parameters such as viscosity, electrical conductivity and their interdependence is needed for better understanding of the reason for such behavior.

Both XRD and FT-IR analyses confirm the incorporation of HA in the composite electrospun mats. Based on literature and the results obtained from degradation studies, a schematic of the progress of degradation is shown in (Figure 10). As expected, lowering the MW has increased the degradation rate. Additionally the presence of HA has also accelerated the degradation. This can be attributed to the hydrophilic nature of $\mathrm{HA}$, which facilitates easier and quicker penetration of water into the composite, consequently accelerating the hydrolysis reaction that occurs during PCL degradation [2]. Similar effect of HA in a PCL matrix during degradation has been reported [17].

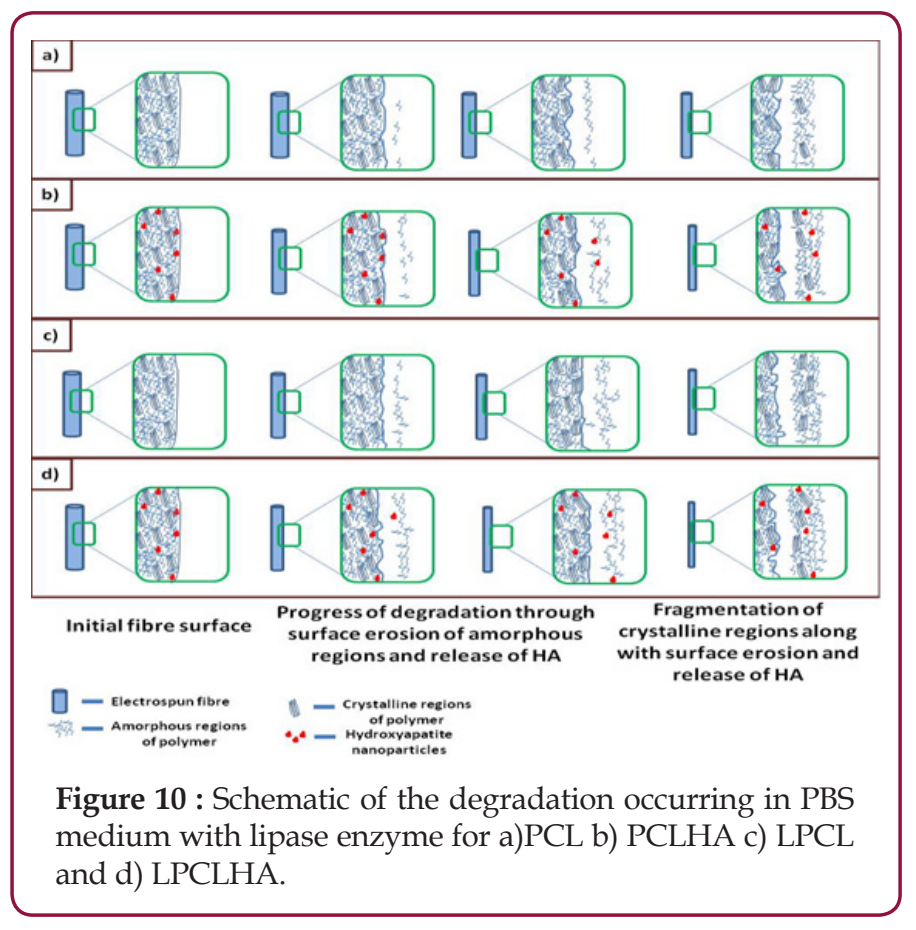

Since degradation of PCL proceeds through surface erosion, there is also the possibility of the gradual release of HA nanoparticles that are embedded in the composite mats. Thus release of HA is also contributing to weight loss. Also since PCL degrades slower than LPCL, the release of HA from PCLHA may have also occurred later than that of LPCLHA. This is seen (Figure 5a), where weight loss in PCLHA overtakes LPCL, due to the onset of release of HA after 2nd week. The faster degrading low Mw PCL in LPCL and the presence of HA in PCLHA had accelerated the degradation and resulted in 
their structural disintegration by 5 th week. Both the mentioned factors were present in LPCLHA which resulted in it degrading and losing its structural integrity earlier by 4 th week.

Thus the faster degrading nature along with the presence of HA could have resulted in LPCLHA showing the highest cell metabolic activity and widespread cell adhesion and proliferation (Figure 10). Rapid disintegration of polymers has the tendency to induce inflammatory reactions due to the acidic by-products [18]. Although slow degrading PCL has not been reported to cause any such major issues, this issue may arise with the relatively faster degrading LPCL. However the addition of HA to LPCL seems beneficial due to their buffering action [7]. As seen in (Figure 5), even though the LPCLHA has the highest degradation rate, the corresponding decrease in $\mathrm{pH}$ is the lesser than the polymer mats. This also seems to be true with PCLHA.

\section{Conclusion}

It was hypothesized that, if chain entanglements necessary for Electrospinning were achieved, even low MW PCL can be electrospun. Though this was possible with the 45PCL, with 10PCL Electrospinning was not possible even at high solute concentrations. The 45PCLHA was found to degrade faster in PBS buffer than the 45PCL, 80PCL and 80PCLHA mats. The electrospun mats were found to be cytocompatible and suitable for growth of osteoblast like cells. Hence investigations on the Electrospinning of low MW varieties of PCL can result in widening the scope of applications of PCL and PCL based composite scaffolds in bone tissue engineering.

\section{References}

1. Chen EC, Wu TM (2007) Isothermal crystallization kinetics and thermal behavior of poly ( $\varepsilon$-caprolactone)/multi-walled carbon nanotube composites. Polym Degrad Stab 92(6): 1009-1015.

2. Lam CX, Savalani MM, Teoh SH, Hutmacher DW (2008) Dynamics of in vitro polymer degradation of polycaprolactone-based scaffolds: accelerated versus simulated physiological conditions. Biomed Mater 3(3): 34108.

3. Abedalwafa M, Wang F, Wang L, Li C (2013) Biodegradable poly-epsiloncaprolactone (PCL) for tissue engineering applications : a review. Rev Adv Mater Sci 34(2): 123-140.

4. Sheikh Z, Najeeb S, Khurshid Z, Verma V, Rashid H, et al. (2015) Biodegradable materials for bone repair and tissue engineering applications. Materials 8(9): 5744-5794.
5. Venkatesan YC, Sampath Kumar TS, Raj DK, Kumary TV (2017) Electrospun cytocompatible polycaprolactone blend composite with enhanced wettability for bone tissue engineering. J Nanosci Nanotechnol 17(4): 2320-2328.

6. Ma Z, He W, Yong T, Ramakrishna S (2005) Grafting of gelatin on electrospun poly (caprolactone) nanofibers to improve endothelial Cell spreading and proliferation and to control cell orientation. Tissue Eng 11(7,8): 1149-1158.

7. Webster TJ (2010) Mechanical properties of dispersed ceramic nanoparticles in polymer composites for orthopedic applications. Int J Nanomedicine 5: 299-313.

8. Woodruff MA, Hutmacher DW (2010) the return of a forgotten polymerpolycaprolactone in the 21st century. Prog Polym Sci 35(10): 1217-1256.

9. Sun H, Mei L, Song C, Cui X, Wang P (2006) The in vivo degradation, absorption and excretion of PCL-based implant. Biomaterials 27(9): 1735-1740.

10. Woodward SC, Brewer PS, Moatamed F, Schindler A, Pitt CG (1985) The intracellular degradation of poly ( $\varepsilon$-caprolactone). J Biomed Mater Res 19(4): 437-444.

11. Hsu CM (2003) Electrospinning of Poly ( $\varepsilon$-Caprolactone). MS Thesis Worcester Polytechnic Institute.

12.Zhang J, Liu H, Xu H, Ding JX, Zhuang XL, et al. (2014) Molecular weight-modulated electrospun poly( $\varepsilon$-caprolactone) membranes for postoperative adhesion prevention. RSC Adv 4 (79): 41696-41704.

13. Kenawy ER, Layman JM, Watkins JR, Bowlin GL, Matthews JA, et al. (2003) Electrospinning of poly(ethylene-co-vinyl alcohol) fibers. Biomaterials 24(6): 907-913.

14. Shenoy SL, Bates WD, Frisch HL, Wnek GE (2005) Role of chain entanglements on fiber formation during electrospinning of polymer solutions: good solvent, non-specific polymer? Polymer interaction limit. Polymer 46(10): 3372-3384.

15. Rameshbabu N, Rao KP, Sampath Kumar TS (2005) Acclerated microwave processing of nanocrystalline hydroxyapatite. J Mater Sci 40(23): 6319-6323.

16. Catledge SA, Clem WC, Shrikishen N, Chowdhury S, Stanishevsky AV, et al. (2007) An electrospun triphasic nanofibrous scaffold for bone tissue engineering. Biomed Mater 2(2): 142-150.

17. Ang KC, Leong KF, Chua CK, Chandrasekaran M (2007) Compressive properties and degradability of poly ( $\varepsilon$-caprolatone)/hydroxyapatite composites under accelerated hydrolytic degradation. J Biomed Mater Res Part A 80 A(3): 655-660.

18. Agrawal CM, Athanasiou KA (1997) Technique to control pH in vicinity of biodegrading PLA-PGA implants. J Biomed Mater Res 38(2): 105-114.

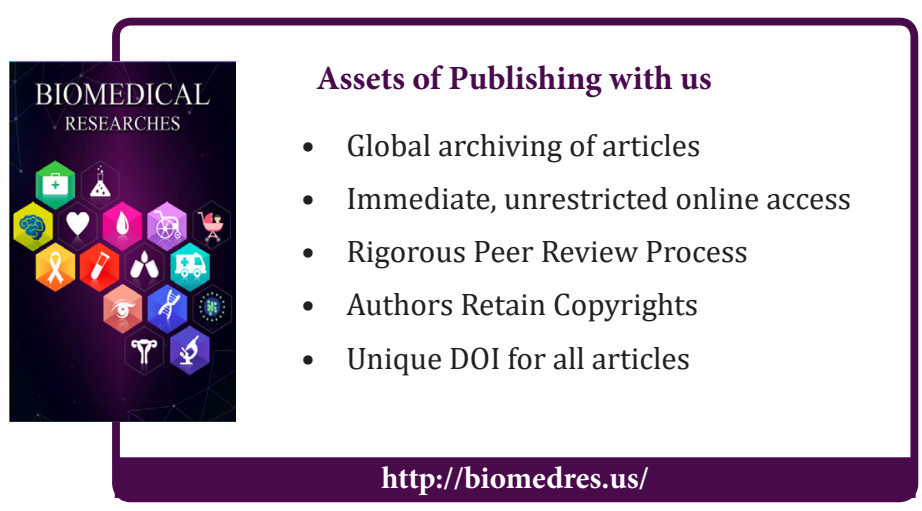

